\title{
Poor appetite and overeating reported by adults in Australia during the coronavirus-19 disease pandemic: a population-based study
}

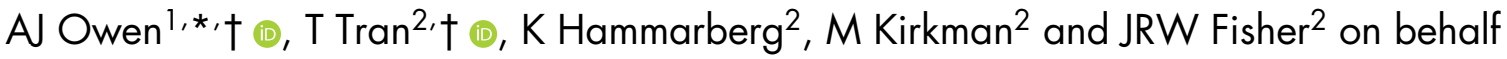 \\ of the COVID-19 Restrictions Impact Research Group \\ ${ }^{1}$ Centre of Cardiovascular Research and Education in Therapeutics, School of Public Health \& Preventive Medicine, \\ 553 St Kilda Rd, Melbourne, Victoria 3004, Australia: ${ }^{2}$ Global and Women's Health, School of Public Health and \\ Preventive Medicine, Monash University, Melbourne, Victoria, Australia
}

Submitted 8 July 2020: Final revision received 2 September 2020: Accepted 21 September 2020: First published online 25 September 2020

\begin{abstract}
Objective: As a result of the coronavirus-19 disease (COVID-19) pandemic, Australia adopted emergency measures on 22 March 2020. This study reports the effect of the COVID-19 lockdown on appetite and overeating in Australian adults during the first month of emergency measures.

Design: This study reports analysis of data from the population-based, selfcompleted survey. The main outcome measure was an item from the Patient Health Questionnaire 9 asking: 'Over the past 2 weeks, how often have you been bothered by poor appetite or overeating?'. Data on sociodemographic factors, symptoms of anxiety and depression, and the impact of COVID-19 and lockdown were also collected. Multivariable logistic regression was used to examine associations with poor appetite or overeating.

Setting: An anonymous online survey available from 3 April to 2 May 2020.

Participants: A total of 13829 Australian residents aged 18 years or over.

Results: The weighted prevalence of being bothered by poor appetite or overeating in the past 2 weeks was $53.6 \%$, with $11.6 \%(95 \%$ CI 10.6, 12.6) of the cohort reporting poor appetite or overeating nearly every day. High levels of anxiety, concern about contracting COVID-19, being in lockdown with children and reporting a severe impact of the lockdown were associated with increased odds of poor appetite or overeating.

Conclusions: Given the widespread prevalence of being bothered by poor appetite or overeating, universal public health interventions to address emotionfocused or situational eating during periods of lockdown may be appropriate.
\end{abstract}

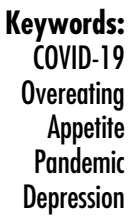

Keywords:

COVID-19

Appetite

Depression
The rapidly escalating crisis associated with the coronavirus19 disease (COVID-19) pandemic arising from severe acute respiratory syndrome coronavirus- 2 infection led to a declaration of a state of emergency in Australia on 22 March 2020. All non-essential activity was halted, and people were required by law to stay at home, with the exception of shopping for food and essential items, medical care, work (if unable to work remotely) and limited daily outdoor exercise. Physical distancing measures were required outside the home, and visiting family and friends was prohibited (except for providing essential care).

The first two authors are joint first author.
By the start of May 2020, Australia had experienced a limited spread of the virus causing COVID-19, with fewer than 7000 cases in total (280 cases per million population $)^{(1)}$. The lockdown has had profound social and personal effects, including widespread unemployment and its financial ramifications, disruption to social interaction and concern about contracting a potentially fatal new disease. Evidence of the profound impact of COVID-19 and its associated restrictions on the mental health of people around the world is emerging ${ }^{(2,3)}$, but there is considerable variation in the approaches being taken to manage COVID-19 in countries with different World Bank Income Classifications and transmission levels ${ }^{(4)}$. 
Diet is a key driver of health ${ }^{(5)}$ and well-being ${ }^{(6,7)}$, not only through the provision of nutrients which support health (including immune health) ${ }^{(8)}$ or patterns of consumption which influence disease risk $^{(5)}$, but also the social health benefits of shared meals ${ }^{(9)}$. In other countries, the pandemic and associated containment strategies are thought to have affected eating behaviour. In Italy, which experienced a severe outbreak early in the pandemic, one survey found that around $53 \%$ of respondents reported eating more during lockdown and 19.5\% reported weight gain ${ }^{(10)}$. Another survey of adolescents and adults in Italy found that over half reported appetite changes, with some evidence that this varied by age, gender and work status ${ }^{(11)}$. In a survey of adults in Poland, more than $40 \%$ reported eating more and $42 \%$ reported weight change (loss or gain) ${ }^{(12)}$. In China (including sampling in Hubei province, which is thought to be the origin of SARS-CoV-2), dietary diversity was found to be lower in more affected regions ${ }^{(13)}$. Aside from the society-wide impact of confinement on diet, loss of the sense of taste and smell is a common side effect in those who contract COVID-19 ${ }^{(14)}$; this may also affect dietary intake.

The objective of this study was to identify self-reports of being bothered by poor appetite or overeating in adults in Australia during the COVID-19 lockdown and learn whether this differs by age, gender or personal and living circumstances. The goal was to contribute to more effective targeting of interventions to improve nutrition and health for those at-risk.

\section{Methods}

This paper reports results of a short, anonymously completed, self-report survey of people living in Australia and aged at least 18 years, with data collected from 3 April 2020 to 2 May 2020. The questionnaire included sociodemographic questions, fixed-response-option questions about the experiences of living with the COVID19 pandemic and resulting restrictions, and two widely used standardised psychometric instruments of symptoms of depression and anxiety. The survey methods have been published ${ }^{(2)}$

Symptoms of anxiety were assessed using the Generalised Anxiety Disorder Scale- $7^{(15)}$. The Generalised Anxiety Disorder Scale-7 is a seven-item validated scale assessing frequency of experiencing common symptoms of anxiety in the preceding 2 weeks. Scores of 5-9 represent mild anxiety, 10-14 moderate anxiety and 15-21 severe anxiety. Clinically significant anxiety is defined as a Generalised Anxiety Disorder Scale-7 score $\geq 10$.

Symptoms of depression were assessed using the Patient Health Questionnaire $9^{(16)}$, a nine-item validated scale assessing frequency of experiencing common symptoms of depression in the preceding 2 weeks. Aggregated responses to the nine items provide an indicative scale of depression symptom severity. Patient Health Questionnaire 9 scores of 5-9 represent mild, 10-14 moderate, 15-19 moderately severe and $\geq 20$ severe depressive symptoms. Clinically significant symptoms of depression were defined as Patient Health Questionnaire 9 scores $\geq 10$.

Bothersome poor appetite or overeating was determined from item 5 of the Patient Health Questionnaire $9^{(16)}$, which asks respondents 'Over the past 2 weeks, how often have you been bothered by poor appetite or overeating?' with response options of 'not at all', 'several days', 'more than half the days' and 'nearly every day'.

\section{Sociodemographic characteristics}

The survey collected information on age, gender, postcode, whether born in Australia, living circumstances (who do you live with?) and occupation. Occupation was collected using ten response items (see Ref. (2)); for the purpose of this analysis, they have been collapsed into four groups: 'Continuing paid work or study from home', 'Work or study has been stopped by COVID-19', 'Leaving the home to go to work' and 'Unpaid caring, welfare benefits, or fixed-income'. This last group included those who reported that they were unpaid carers of children or dependent relatives, retired, had government benefits as their main source of income support or were unemployed before the COVID-19 pandemic. To obtain area-level socio-economic status, the Australian Bureau of Statistics Socio-economic Indices for Areas ${ }^{(17)}$ were derived from a respondent's postcode, from which quintiles were developed.

Personal impact of COVID-19 restrictions was assessed by the question 'How badly have the COVID-19 restrictions affected your daily life?' and rated on a scale of $0-10$ ('not at all' to 'very badly'). A high adverse impact of restrictions was defined as a score $\geq 8$. Anxiety relating to contracting COVID-19 was assessed by the question 'How worried are you that you will catch COVID-19?' and rated on a scale of 0-10 (from 'not at all worried' to 'extremely worried'). Being highly worried about contracting COVID-19 was defined as a score $\geq 8$. Personal experience of COVID-19 (being treated or tested for COVID-19, or knowing someone who has contracted COVID-19) was also assessed.

\section{Statistical analysis}

Population prevalence and $95 \%$ CI of people bothered by poor appetite or overeating was estimated, adjusting for differences in sociodemographic characteristics between the sample and the Australian population ${ }^{(17)}$. The adjustment was made using post-stratification weights ${ }^{(2)}$.

Multivariable-adjusted logistic regression modelling of factors associated with being bothered by poor appetite or overeating in the previous 2 weeks was 
undertaken. The factors included in the model were sociodemographic characteristics, living circumstances, mental health status and experiences of COVID-19 and associated restrictions.

Data analyses were conducted using STATA version 16 (StataCorp.) with only complete data included.

Table 1 Sociodemographic characteristics of the study sample

\begin{tabular}{lrr}
\hline & $n$ & $\%$ \\
\hline Total respondents & 13829 & \\
Gender & 10434 & $75 \cdot 5$ \\
$\quad$ Female & 3328 & $24 \cdot 1$ \\
Male & 67 & 0.5 \\
Other & & \\
Age group in years & 1337 & $9 \cdot 7$ \\
18-29 & 2294 & $16 \cdot 6$ \\
30-39 & 2854 & $20 \cdot 6$ \\
$40-49$ & 3064 & $22 \cdot 2$ \\
$50-59$ & 2833 & 20.5 \\
$60-69$ & 1447 & 10.5 \\
$70+$ & 2147 & 15.5 \\
Any experience of COVID-19 (whether the & &
\end{tabular}

Table 2 Prevalence of poor appetite or overeating coronavirus-19 disease (COVID-19) lockdown in Australia

Over the past 2 weeks, how often have you been bothered by poor appetite or overeating?

Prevalence (\%) with weighted data $95 \% \mathrm{Cl}$

\begin{tabular}{lll}
\hline Not at all & 46.4 & $44.9,48 \cdot 0$ \\
Several days & $25 \cdot 8$ & $24.5,27 \cdot 2$ \\
More than half of the days & $16 \cdot 2$ & $15 \cdot 0,17.5$ \\
Nearly every day & 11.6 & $10.6,12.6$
\end{tabular}

Prevalence (\%) weighted by State, Socio-economic Indices for Areas decile (socioeconomic area) gender and age.

\section{Results}

A total of 13829 adults (mean age 50.4 (SD 14.9) years) contributed complete data. Their demographic characteristics are presented in Table 1. The weighted prevalence of being bothered at all by poor appetite or overeating in the previous 2 weeks was $53.6 \%$, with $11.6 \%$ (95\% CI $10 \cdot 6,12 \cdot 6)$ of respondents bothered by poor appetite or overeating nearly every day (Table 2).

Multiple logistic regression analysis indicated that age, gender, socio-economic status, living circumstances, being born overseas, concern about contracting COVID-19 and

tested for COVID-19, or lived with or knew someone with COVID-19)

Highly worried about contracting COVID-19 (scale score $\geq 8$ )

Median

Range

High adverse impact of restrictions (scale score $\geq 8$ )

Median

Range

State

New South Wales

Australian Capital Territory

Victoria

Queensland

South Australia

Western Australia

Tasmania

Northern Territory

Living in a major city

SEIFA quintiles

Quintile 1 (lowest socio-economic area)

Quintile 2

Quintile 3

Quintile 4

Quintile 5 (highest socio-economic area)

Living situation

On your own

With your partner and children

With children and without a partner

With adult family members

In a shared house with non-family members Other

Born overseas

Occupation

Continuing paid work or study from home

Work or study has been stopped by COVID

Leaving the house to work

Unpaid caring, welfare benefits, fixed-income
With only your partner $13 \cdot 9$ $13 \cdot 1-14 \cdot 8$

$25 \cdot 2$ 23.8-26.8

$2753 \quad 19.9$

$\begin{array}{ll}465 & 3.4\end{array}$

$6105 \quad 44.1$

$1939 \quad 14.0$

$836 \quad 6.0$

$1177 \quad 8.5$

$445 \quad 3.2$

$\begin{array}{ll}109 & 0.8\end{array}$

$9045 \quad 65.4$

$1093 \quad 7.9$

$1541 \quad 11.1$

$2228 \quad 16 \cdot 1$

$3038 \quad 22.0$

$5929 \quad 42.9$

$4203 \quad 30 \cdot 4$

$2660 \quad 19.2$

$3875 \quad 28.0$

$578 \quad 4.2$

$1552 \quad 11.2$

$616 \quad 4.5$

$345 \quad 2.5$

$\begin{array}{ll}3150 & 22.8\end{array}$

$6767 \quad 49 \cdot 6$

$887 \quad 6.5$

$2262 \quad 16.6$

$3730 \quad 27 \cdot 3$

COVID-19, coronavirus-19 disease; SEIFA, Socio-economic Indices for Areas. Values given as $n(\%)$, except for 'Highly worried about contracting COVID-19' and 'High adverse impact of restrictions' which are given as prevalence (\%) weighted for State, SEIFA decile, gender and age. being highly affected by the COVID-19 pandemic restrictions were associated with odds of being bothered by poor appetite or overeating (Table 3). Above the age of 40 years, after multivariate adjustment, increasing age was incrementally associated with decreasing odds of being bothered by poor appetite or overeating in the previous fortnight.

Being male was associated with lower odds of being bothered by poor appetite or overeating, as was high socio-economic advantage (Table 3). Those living alone, with children and without a partner, or in a shared house with non-family members had significantly greater odds of being bothered by poor appetite or overeating than people living in a household with a partner and no children (Table 3).

Being in unpaid caring roles, unemployed before the COVID pandemic and still unemployed, retired or on government welfare benefits were associated with slightly lowered odds of being bothered by poor appetite or overeating compared with those who were continuing paid work or study from home.

\section{COVID-19 impact, well-being and being botbered by poor appetite or overeating}

After adjustment for sociodemographic factors, anxiety and depression, respondents who were highly concerned about contracting COVID-19 and those who felt a high level of adverse impact of the pandemic restrictions had significantly increased odds of being bothered by poor appetite or overeating (Table 3 ). There was only a small number of respondents who had contracted COVID-19 ( $n$ 56), which limits the ability to compare this group to the wider sample. 
Table 3 Multivariable-adjusted logistic regression model of factors associated with being bothered by poor appetite or overeating in the past 2 weeks

\begin{tabular}{|c|c|c|c|c|}
\hline Gender & OR & $P$-value & \multicolumn{2}{|c|}{$95 \% \mathrm{Cl}$} \\
\hline Female & Rof & & & \\
\hline Male & 0.67 & $<0.001$ & 0.61 & 0.74 \\
\hline Other & 0.45 & 0.016 & 0.23 & 0.86 \\
\hline \multicolumn{5}{|l|}{ Age group (years) } \\
\hline $18-29$ & Ref. & & & \\
\hline $30-39$ & 0.92 & 0.349 & 0.77 & 1.10 \\
\hline $40-49$ & 0.76 & 0.003 & 0.64 & 0.91 \\
\hline $50-59$ & 0.64 & $<0.001$ & 0.54 & 0.76 \\
\hline $60-69$ & 0.49 & $<0.001$ & 0.41 & 0.58 \\
\hline $70+$ & 0.34 & $<0.001$ & 0.27 & 0.42 \\
\hline $\begin{array}{l}\text { Moderate, moderately severe or severe (clinically significant) depressive } \\
\text { symptoms, PHQ-9 score } \geq 10 \text { ) }\end{array}$ & $10 \cdot 58$ & $<0.001$ & $9 \cdot 12$ & $12 \cdot 27$ \\
\hline $\begin{array}{l}\text { Moderate, or severe anxiety (clinically significant) symptoms of anxiety, } \\
\text { GAD-7 score } \geq 10 \text { ) }\end{array}$ & $1 \cdot 88$ & $<0.001$ & $1 \cdot 62$ & $2 \cdot 18$ \\
\hline $\begin{array}{l}\text { Any experience of COVID-19 (whether the respondent had been diagnosed } \\
\text { with or tested for COVID-19, or lived with or knew someone with COVID-19) }\end{array}$ & 1.07 & 0.244 & 0.96 & $1 \cdot 19$ \\
\hline Highly worried about contracting COVID-19 (scale score $>8$ ) & 1.27 & $<0.001$ & $1 \cdot 13$ & 1.42 \\
\hline High adverse impact of restrictions (scale score $\geq 8$ ) & $1 \cdot 30$ & $<0.001$ & $1 \cdot 18$ & 1.44 \\
\hline \multicolumn{5}{|l|}{ 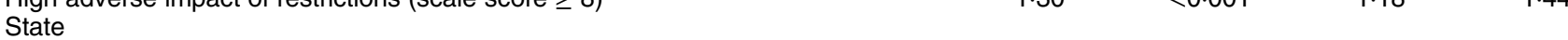 } \\
\hline New South Wales & Ref. & & & \\
\hline Australian Capital Territory & 1.03 & 0.792 & 0.82 & 1.30 \\
\hline Victoria & 1.00 & 0.998 & 0.90 & 1.11 \\
\hline Queensland & 1.09 & 0.239 & 0.95 & 1.25 \\
\hline South Australia & $1 \cdot 19$ & 0.057 & 0.99 & 1.43 \\
\hline Western Australia & 1.03 & 0.698 & 0.88 & 1.21 \\
\hline Tasmania & $1 \cdot 10$ & 0.433 & 0.86 & 1.41 \\
\hline Northern Territory & 0.99 & 0.960 & 0.64 & 1.53 \\
\hline Major city & $1 \cdot 16$ & 0.007 & 1.04 & 1.29 \\
\hline \multicolumn{5}{|l|}{ SEIFA quintiles } \\
\hline Quintile 1 (Lowest socio-economic area) & Ref. & & & \\
\hline Quintile 2 & 1.00 & 0.969 & 0.83 & 1.20 \\
\hline Quintile 3 & 0.96 & 0.626 & 0.81 & 1.14 \\
\hline Quintile 4 & 0.86 & 0.080 & 0.72 & 1.02 \\
\hline Quintile 5 (Highest socio-economic area) & 0.78 & 0.004 & 0.65 & 0.92 \\
\hline \multicolumn{5}{|l|}{ Living situation } \\
\hline With only your partner & Ref. & & & \\
\hline On your own & 1.38 & $<0.001$ & 1.23 & 1.54 \\
\hline With your partner and children & 1.19 & 0.003 & 1.06 & 1.33 \\
\hline With children and without a partner & 1.48 & $<0.001$ & $1 \cdot 20$ & 1.83 \\
\hline With adult family members & $1 \cdot 11$ & 0.166 & 0.96 & 1.28 \\
\hline In a shared house with non-family members & 1.62 & $<0.001$ & $1 \cdot 30$ & 2.02 \\
\hline Other & $1 \cdot 12$ & 0.399 & 0.86 & 1.46 \\
\hline Born overseas & 0.80 & $<0.001$ & 0.73 & 0.88 \\
\hline \multicolumn{5}{|l|}{ Occupation } \\
\hline Continuing paid work or study from home & Ref. & & & \\
\hline Work or study stopped by COVID-19 & 1.01 & 0.900 & 0.85 & 1.20 \\
\hline Leaving the house to work & 0.97 & 0.583 & 0.87 & 1.08 \\
\hline Unpaid caring, welfare benefits, fixed-income & 0.82 & 0.001 & 0.73 & 0.92 \\
\hline
\end{tabular}

COVID-19, coronavirus-19 disease; GAD-7, Generalised Anxiety Disorder Scale; SEIFA, Socio-economic Indices for Areas; Ref., reference category.

For each variable examined in the model, the given OR are adjusted for all remaining covariates: age, gender, SEIFA, state, residential area classification (major city $v$. outside major cities), being born overseas, living circumstance, occupation, anxiety, depression, concern about contracting COVID-19, felt impact of restrictions, personal experience of COVID-19.

Clinically significant anxiety and depression scores $(\geq 10)$ were each associated with increased odds of being bothered by poor appetite or overeating (Table 3).

\section{Discussion}

These findings demonstrate that, in the first month of COVID-19 pandemic restrictions, more than half of the sampled adults reported that they were bothered by poorer appetite or eating more than usual. Women, those living alone, those living with children and without a partner, those very worried about contacting COVID-19 and those who felt a greater adverse effect of pandemic restrictions, were particularly likely to report being bothered by poor appetite or overeating. Older age, living with adult family members without children, higher socio-economic status, not being in paid work or study before COVID-19 and living in a regional area appeared to reduce the risk of being bothered by appetite and overeating during the pandemic. 
Clinically significant depression and anxiety were also associated with substantially increased odds of reporting being bothered by poor appetite or overeating.

Emotional eating (eating in response to negative emotions) is a well-described phenomenon ${ }^{(18)}$ and has been implicated in the pathway between depression and obesity $^{(19)}$. Emotional eating is most commonly reported in response to boredom, loneliness and anxiety ${ }^{(20)}$. Stress can suppress appetite ${ }^{(21)}$, and loss of appetite is a common feature of depression and anxiety ${ }^{(22)}$.

Living alone, living with children or living in shared accommodation with non-family members were all associated with increased odds of being bothered by poor appetite or overeating, compared with living with a partner. Eating alone is a known risk factor for malnutrition in older adults $^{(23)}$, and eating either alone or with non-family members is associated with increased risk of obesity when compared with eating with family ${ }^{(24)}$. At the time of the survey, schools were shut to all but the children of essential workers, and most households with children, were 'learning from home'. While those with sole responsibility for children were most bothered by poor appetite or overeating, those living with a partner and children were also more likely to be bothered by poor appetite or overeating than those living with a partner only. This suggests the possibilities that having children at home during the lockdown may induce stress which affects appetite or overeating or that catering to the dietary needs of children in the home may present additional eating opportunities. Living outside major metropolitan cities was associated with lower odds of being bothered by poor appetite or overeating. Possible explanations for this regional resilience may include feeling somewhat removed from the epicentre of the pandemic (which was concentrated at the time in major cities ${ }^{(1)}$ ), or perhaps having working and daily lives that were less disrupted by lockdown.

There were important age and gender differences in the reports of being bothered by poor appetite or overeating. Women were more likely to report being bothered by poor appetite or overeating than men. This is consistent with evidence that women tend to report greater weight/body shape concerns, eating concerns and binge eating than men ${ }^{(25)}$. Increasing age was associated with lower odds of being bothered by poor appetite or overeating, despite older age being associated with more worry about contracting COVID-19 ${ }^{(2)}$. Possible explanations for this agerelated resilience to eating behaviour change may be more habitually stable eating patterns ${ }^{(26)}$, age-related decline in concern about body shape or weight change ${ }^{(27)}$, being more financially secure or having greater life experience of difficult situations and thus more capacity to regulate emotions in the face of the life changes imposed by lockdown. In young adult men, higher anxiety has been reported to be associated with uncontrolled eating, but uncontrolled eating also decreased with age ${ }^{(22)}$. However, a similar pattern of age-related decline in disordered eating was not noted in young women ${ }^{(22)}$.

Environmental factors are known to be associated with food consumption. Overeating is related to the presence of palatable food in the environment ${ }^{(28)}$; with a pandemicrelated lockdown, some people may have found themselves working from home in close proximity to the kitchen and its temptations. Those who were not working or studying outside the home before lockdown (and perhaps more accustomed to spending time at home) appeared to fare better in not being bothered by dietary behaviour, even though this group included people on low or fixed incomes (e.g. pensions), a population group generally considered to be at increased dietary risk ${ }^{(29)}$. In a US study of economically disadvantaged women, resistance to emotional eating and favourable social eating behaviours were associated with better diet quality and lower BMI, with evidence that these factors were key to mediating the relationship between food insecurity and BMI in women living on low incomes ${ }^{(30)}$. A feature of the early stages of the first pandemic wave lockdown in Australia was panic-buying or stockpiling of long-life foods such as flour, sugar, dried pasta, rice, biscuits, and bottled and canned foods. Baking and home cooking (notably 'iso-baking') were also prominent during this period ${ }^{(31)}$ and may have contributed to an increase in the available food in the home environment.

There appeared to be a socio-economic gradient in these findings, with those of greatest advantage least bothered by poor appetite or overeating, although following adjustment for other factors only the two extreme quintiles of socio-economic area status remained significantly different. The ability of economic advantage to buffer against any personal economic stress arising from the pandemic lockdown (e.g. losing or being stood down from employment, business downturn) may be one explanation. Greater access to health and well-being services and preventive healthcare may be another.

These findings of adult eating behaviour concerns in Australia during the early stages of the COVID-19 pandemic do bear some similarities to what has been observed in other countries. The proportion of those who reported being bothered by poor appetite or overeating in this study was similar to self-reported dietary behaviours in Italy ${ }^{(10)}$ and Poland ${ }^{(12)}$. In the survey of 1932 Italians, $53 \%$ reported that they were eating more during confinement; $42 \%$ of survey respondents attributed their dietary change to stress, anxiety or boredom ${ }^{(10)}$. Another survey covering Northern, Central and Southern Italy found that $17.8 \%$ of the sample reported reduced appetite, while $34.4 \%$ reported increased appetite during the COVID-19 lockdown ${ }^{(11)}$.

\section{Strengths and limitations}

A strength of this study is the large sample, with respondents from every state and territory of Australia, and the 
sample weighting to enhance national representativeness. Australia is a high-income country with a universal health and welfare system, and thus the findings of this study may not be directly applicable in other settings, including lowincome countries ${ }^{(32)}$. Validated measures of symptoms of anxiety and depression were employed, and the survey included a question which captured level of concern about contracting COVID-19 as well as a question about the impact of the pandemic restrictions. It should be noted that the question about eating behaviour asked whether respondents were 'bothered by poor appetite or overeating', and does not enable these two eating behaviour constructs to be considered separately. It was not possible to determine the magnitude of any change in eating behaviour, not to determine whether consequences of this bothersome eating behaviour had manifested. It should also be noted that analysis of the association between depression scores and poor appetite or overeating is limited by the fact that the appetite question is a component of the score; thus, the OR for this should be considered with caution.

\section{Implications and conclusions}

The findings of this study indicate that bothersome poor appetite or overeating was experienced by over $50 \%$ of adults during the first month of COVID-19 restrictions during the first wave of the pandemic. While short periods of dietary behaviour change may not significantly impact upon weight, with longer periods of lockdown, this may become of greater public health concern. Prolonged overeating increases risk of weight gain and obesity, with obesity being a wellknown risk factor for chronic disease ${ }^{(33)}$. Weight gain may also cause psychological distress associated with unwanted changes in body shape ${ }^{(25)}$. During the first wave of the pandemic, research has emerged indicating that obesity is a risk factor for greater severity of COVID-19 ${ }^{(34)}$. Weight gain might therefore heighten distress among those already concerned about contracting COVID-19. With subsequent waves of the pandemic and variability in responses to these across different countries, longitudinal studies of eating behaviour and other behavioural risk factors are needed to understand both short-term and long-term mental and physical health impacts $^{(35)}$.

There are factors that may limit (older age, regional dwelling, being more accustomed to spending time at home) or increase (female gender, anxiety, depression, being in lockdown with children) risk of being bothered by poor appetite or overeating for adults living in a high-income country during lockdown necessitated to control a pandemic. From a public health perspective, this research can inform universal and targeted public health promotion strategies to assist people to restore healthier eating patterns during periods of societywide confinement and disruptions to routine. It has been suggested that cognitive behavioural therapy may be one option for improving emotional regulation and assisting development of coping strategies associated with lockdown, and e-health delivery of cognitive behavioural therapy may be appropriate for those experiencing severe distress ${ }^{(36)}$. As more than half of adults in this large sample reported being bothered by poor appetite or overeating, population-wide public health strategies may also be warranted for subsequent pandemic waves and periods of lockdown.

\section{Acknowledgements}

Acknowledgements: We thank the many thousands of study participants for their contribution. The feedback from participants in this survey has been crucial in guiding development of future research. We would like to acknowledge other members of the COVID-19 Restrictions Impact Research Group: Jayagowri Sastry, Hau Nguyen, Heather Rowe, Sally Popplestone, Ruby Stocker and Claire Stubber. Financial support: This research was supported by a generous untied philanthropic donation from Professor John McBain and Dr Penny Foster. J.F. is supported by the Finkel Professorial Fellowship which is funded by the Finkel Family Foundation, and T.T. is supported by a Monash University Strategic Bridging Fellowship. Conflict of interest: There are no conflicts of interest. Authorship: A.J.O. was involved in the development of the analysis plan, drafted the initial manuscript and reviewed and revised the manuscript. T.T. led development of the analytical plan, undertook statistical analyses and reviewed and revised the manuscript. K.H., M.K. and J.R.W.F. conceptualised the study, designed the data collection instruments and critically reviewed the manuscript for important intellectual content. All authors approved the final manuscript as submitted and are accountable for this work. Ethics of buman subject participation: This study was conducted according to the guidelines laid down in the Declaration of Helsinki, and all procedures involving human participants were approved by the Monash University Human Research Ethics Committee (2020-24080-42716). As this was an anonymous online survey, consent was inferred by willingness to complete the survey.

\section{References}

1. Australian Government (2020) Coronavirus (COVID-19) current situation and case numbers. https://www.health.gov. $\mathrm{au} /$ news/health-alerts/novel-coronavirus-2019-ncov-healthalert/coronavirus-covid-19-current-situation-and-case-numbers (accessed August 2020).

2. Fisher JRW, Tran TD, Hammarberg K et al. (2020) Mental health of people in Australia in the first month of COVID19 restrictions: a national survey. Med J Aust. https://www. mja.com.au/journal/2020/mental-health-people-australia-firstmonth-covid-19-restrictions-national-survey (Pre-print 10 June 2020).

3. Hamel L, Lopes L, Muñana C et al. (2020) Coronavirus Poll: March 2020. https://www.kff.org/coronavirus-covid-19/ poll-finding/kff-coronavirus-poll-march-2020/ (accessed June 2020). 
4. Tran BX, Ha GH, Nguyen LH et al. (2020) Studies of novel coronavirus disease 19 (COVID-19) pandemic: a global analysis of literature. Int J Env Res Pub Health 17, 4095 .

5. Global Burden of Disease Group (2019) Health effects of dietary risks in 195 countries, 1990-2017: a systematic analysis for the Global Burden of Disease Study 2017. Lancet 393 , 1958-1972.

6. Marx W, Moseley G, Berk M et al. (2017) Nutritional psychiatry: the present state of the evidence. Proc Nutr Soc 76, 427-436.

7. Govindaraju T, Sahle BW, McCaffrey TA et al. (2018) Dietary patterns and quality of life in older adults: a systematic review. Nutrients 10, 971.

8. Calder PC, Carr AC, Gombart AF et al. (2020) Optimal nutritional status for a well-functioning immune system is an important factor to protect against viral infections. Nutrients 12, 1181.

9. Fischler C (2011) Commensality, society and culture. Soc Sci Inf 50, 528-548.

10. Scarmozzino F \& Visioli F (2020) Covid-19 and the subsequent lockdown modified dietary habits of almost half the population in an Italian sample. Foods 9, 675 .

11. Di Renzo L, Gualtieri P, Pivari F et al. (2020) Eating habits and lifestyle changes during COVID-19 lockdown: an Italian survey. J Transl Med 18, 229.

12. Sidor A \& Rzymski P (2020) Dietary choices and habits during COVID-19 lockdown: experience from Poland. Nutrients $\mathbf{1 2}$, 1657.

13. Zhao A, Li Z, Ke Y et al. (2020) Dietary diversity among Chinese residents during the COVID-19 outbreak and its associated factors. Nutrients $\mathbf{1 2}, 1699$.

14. Parma V, Ohla K, Veldhuizen MG et al. (2020) More than smell - COVID-19 is associated with severe impairment of smell, taste, and chemesthesis. Chem Senses, June 20, bjaa041.

15. Spitzer RL, Kroenke K, Williams JB et al. (2006) A brief measure for assessing generalized anxiety disorder: the GAD-7. Arch Intern Med 166, 1092-1097.

16. Patten SB \& Schopflocher D (2009) Longitudinal epidemiology of major depression as assessed by the brief Patient Health Questionnaire (PHQ-9). Compr Psychiatry 50, 26-33.

17. Australian Bureau of Statistics 3101.0 - Australian Demographic Statistics (2019). https://www.abs.gov.au/AUSSTATS/abs@. nsf/DetailsPage/3101.0Sep\%202019?OpenDocument. (accessed June 2020).

18. Devonport TJ, Nicholls W \& Fullerton C (2019) A systematic review of the association between emotions and eating behaviour in normal and overweight adult populations. $J$ Health Psychol 24, 3-24.

19. Konttinen H, van Strien T, Männistö S et al. (2019) Depression, emotional eating and long-term weight changes: a population-based prospective study. Int J Behav Nutr Phys Act 16, 28.

20. Wiedemann AA, Ivezaj V \& Barnes RD (2018) Characterizing emotional overeating among patients with and without binge-eating disorder in primary care. Gen Hosp Psychiatry 55, 38-43.
21. Nakamura C, Ishii A, Matsuo T et al. (2020) Neural effects of acute stress on appetite: a magnetoencephalography study. PLoS One 15, e0228039.

22. Aoun C, Nassar L, Soumi S et al. (2019) The cognitive, behavioral, and emotional aspects of eating habits and association with impulsivity, chronotype, anxiety, and depression: a cross-sectional study. Front Behav Neurosci 13, 204.

23. Posner BM, Jette AM, Smith KW et al. (1993) Nutrition and health risks in the elderly: the nutrition screening initiative. Am J Public Health 83, 972-978.

24. Jeong W \& Jang SI (2020) Associations between meal companions and obesity in South Korean adults. Int J Env Res Pub Health 17, 2697.

25. Buchanan NT, Bluestein BM, Nappa AC et al. (2013) Exploring gender differences in body image, eating pathology, and sexual harassment. Body Image 10, 352-360.

26. Sotos-Prieto M, Bhupathiraju SN, Mattei J et al. (2017) Association of changes in diet quality with total and causespecific mortality. New Engl J Med 377, 143-153.

27. Bouzas C, Bibiloni MDM \& Tur JA (2019) Relationship between body image and body weight control in overweight $\geq 55$-year-old adults: a systematic review. Int J Env Res Pub Health 16, 1622.

28. Thomas JG, Doshi S, Crosby RD et al. (2011) Ecological momentary assessment of obesogenic eating behavior: combining person-specific and environmental predictors. Obesity 19, 1574-1579.

29. Laine JE, Baltar VT, Stringhini S et al. (2020) Reducing socioeconomic inequalities in all-cause mortality: a counterfactual mediation approach. Int J Epidemiol 49, 497-510.

30. Sanjeevi N, Freeland-Graves J \& Hersh M (2018) Food insecurity, diet quality and body mass index of women participating in the Supplemental Nutrition Assistance Program: the role of intrapersonal, home environment, community and social factors. Appetite 125, 109-117.

31. Jean Hailes Foundation (2020) Iso-baking 101: keep calm, keep cooking, and carry on. https://www.jeanhailes. org.au/news/healthier-baking-tips-recipes (accessed July 2020).

32. Tran BX, Phan HT, Nguyen TPT et al. (2020) Reaching further by Village Health Collaborators: the informal health taskforce of Vietnam for COVID-19 responses.J Glob Health 10, 010354.

33. GBD 2016 Risk Factors Collaborators (2017) Global, regional, and national comparative risk assessment of 84 behavioural, environmental and occupational, and metabolic risks or clusters of risks, 1990-2016: a systematic analysis for the Global Burden of Disease Study 2016. Lancet 390, $1345-1422$.

34. Lighter J, Phillips M, Hochman S et al. (2020) Obesity in patients younger than 60 years is a risk factor for COVID19 hospital admission. Clin Infect Dis 71, 896-897.

35. Wang C, Pan R, Wan X et al. (2020) A longitudinal study on the mental health of general population during the COVID19 epidemic in China. Brain Behav Immun 87, 40-48.

36. Ho CS, Chee CY \& Ho RC (2020) Mental health strategies to combat the psychological impact of COVID-19 beyond paranoia and panic. Ann Acad Med Singapore 49, 155-160. 